\title{
Nontrivial critical points of asymptotically quadratic functions at resonances
}

\author{
by Michal Fé̌Kan (Bratislava)
}

\begin{abstract}
Asymptotically quadratic functions defined on Hilbert spaces are studied by using some results of the theory of Morse-Conley index. Applications are given to existence of nontrivial weak solutions for asymptotically linear elliptic partial and ordinary differential equations at resonances.
\end{abstract}

1. Introduction. This paper is a continuation of [2] and it is devoted to the study of the existence of nontrivial critical points of asymptotically quadratic functions defined on Hilbert spaces. The paper is divided into three parts.

Section 2 deals with the existence of critical points for functions on infinite-dimensional spaces which are asymptotically quadratic at infinity. Recently several papers dealing with such problems have appeared (see [1, $5,6]$ ). In the first part of Section 2, results on existence of nontrivial critical points are proved for functions with asymptotically linear gradients and whose linear asymptotes are mostly not invertible and trivial critical points are degenerate. Certain relations are crucial between the Morse indices of trivial degenerate critical points and the Morse indices of linear asymptotes. In addition, conditions of the Landesman-Lazer type must be considered (see [7] and [8]). Some theorems of Section 2 are also devoted to a case when there are no such relations, but the given nondegenerate critical points lie on the same level sets. These theorems can be naturally applied when the functions considered are invariant under finite groups of transformations. In that section we also study resonant cases.

Section 3 is devoted to the problem

$$
\begin{aligned}
& -\Delta u+h(u, x)=0, \quad x \in \Omega, \\
& u \mid \partial \Omega=0,
\end{aligned}
$$

1991 Mathematics Subject Classification: 58E05, 34B15, 35D05.

Key words and phrases: weak solutions, boundary value problems, Morse-Conley index. 
where $\Omega$ is an open bounded subset of $\mathbb{R}^{3}$ with a smooth boundary $\partial \Omega$ and $h \in C(\mathbb{R} \times \bar{\Omega}, \mathbb{R})$ is asymptotically linear in $u$ and satisfies $h(0, \cdot)=0$. The existence of a nonzero weak solution of (1.1) is shown.

The last Section 4 deals with the existence of a nonzero solution of the equation

$$
\begin{aligned}
& -y^{\prime \prime}-n^{2} y+g(y)=0, \quad x \in[0, \pi], \\
& y(0)=y(\pi)=0,
\end{aligned}
$$

where $n>1$ is a natural number and $g \in C(\mathbb{R}, \mathbb{R})$ is asymptotically linear satisfying

$$
\lim _{u \rightarrow 0_{ \pm}} \frac{g(u)}{|u|^{m} \operatorname{sgn} u}=l_{ \pm}<\infty
$$

for $m>1$ and $l_{+} l_{-} \geq 0, l_{-}^{2}+l_{+}^{2}>0$. The existence of small solutions of boundary value problems like (1.2) is studied in [3] and [4], where the isolatedness of the zero solution of (1.2) is proved under the condition (1.3).

We study the existence of nonzero solutions in (1.1-2) at resonances and for degenerate cases, i.e. where both asymptotes at infinity and linearizations at zero solutions have nonzero kernels. (1.1) with some symmetries is also investigated.

2. Abstract results. Let $H$ be a Hilbert space with an inner product $\langle\cdot, \cdot\rangle$ and the norm $|\cdot|$. Recall that the Morse index of a bounded self-adjoint linear map is the (finite) dimension of the subspace of all eigenvectors of that map with negative eigenvalues. The Morse index of a critical point of a $C^{2}$-smooth function is the Morse index of its Hessian at that point. The Hessian at a critical point $x$ of a $C^{2}$-smooth function $f$ at $x$ is denoted by Hess $f(x)$. A critical point $x$ of a $C^{1}$-smooth function $f$ is nondegenerate if $f$ is $C^{2}$-smooth at $x$ and Hess $f(x)$ is invertible.

We note that $f \in C^{1}(H, \mathbb{R})$ is said to satisfy the P.S. condition (see $[6$, Remark 4.2]) if every sequence $\left\{u_{i}\right\}_{i=1}^{\infty} \subset H$ such that $\left\{f\left(u_{i}\right)\right\}_{i=1}^{\infty}$ is bounded and $\operatorname{grad} f\left(u_{i}\right) \rightarrow 0$ as $i \rightarrow \infty$ contains a convergent subsequence. It is clear that $f \in C^{1}(H, \mathbb{R})$ satisfies the P.S. condition provided that $\operatorname{grad} f$ is a compact perturbation of a bounded linear, Fredholm, self-adjoint operator and $(\operatorname{grad} f)^{-1}(B)$ is bounded for any bounded subset $B$. The last property is valid if $\operatorname{grad} f$ has an invertible linear asymptote at infinity.

Definition 2.1. Let $f \in C^{1}(H, \mathbb{R})$ have a critical point 0 such that $A=\operatorname{Hess} f(0)$ exists, index $A$ exists and $A$ is Fredholm. $f$ is said to satisfy the condition $\left(C_{+}\right)$, respectively $\left(C_{-}\right)$, if there is a constant $\alpha>0$ such that for any sequences $\left\{r_{n}\right\}_{n=1}^{\infty}, r_{n} \in(0, \infty),\left\{z_{n}\right\}_{n=1}^{\infty}, z_{n} \in H$, satisfying 
$r_{n} \rightarrow 0_{+}$and $\left|z_{n}\right|=O\left(r_{n}^{2}\right)$ as $r_{n} \rightarrow 0_{+}$, we have

$$
\liminf _{n \rightarrow \infty}\left\langle S\left(r_{n} \varphi+z_{n}\right), \varphi\right\rangle / r_{n}^{\alpha}>0
$$

respectively

$$
\limsup _{n \rightarrow \infty}\left\langle S\left(r_{n} \varphi+z_{n}\right), \varphi\right\rangle / r_{n}^{\alpha}<0
$$

uniformly for $\varphi \in \partial B_{1} \equiv\{x \in \operatorname{ker} A|| x \mid=1\}$, where $S=\operatorname{grad} f-A$.

THEOREM 2.2. If $f$ satisfies the condition $\left(C_{+}\right)$, respectively $\left(C_{-}\right)$, then there is a neighbourhood $U$ of 0 and a constant $\varepsilon_{0}>0$ such that the only critical point of $f(x)+\varepsilon|x|^{2} / 2$ in $U$ is 0 for $\varepsilon_{0}>\varepsilon>0$, respectively $-\varepsilon_{0}<$ $\varepsilon<0$.

Proof. We consider the case $\left(C_{+}\right)$, the other one is similar. Assume the contrary. Then there are $\varepsilon_{n} \rightarrow 0_{+}$and $x_{n} \rightarrow 0, x_{n} \neq 0$, such that

$$
\varepsilon_{n} x_{n}+\operatorname{grad} f\left(x_{n}\right)=0 \text {. }
$$

Hence

$$
\begin{aligned}
\varepsilon_{n} x_{n 2}+A x_{n 2}+Q_{A} S\left(x_{n 1}+x_{n 2}\right) & =0, \\
\varepsilon_{n} x_{n 1}+P_{A} S\left(x_{n 1}+x_{n 2}\right) & =0,
\end{aligned}
$$

where $x_{n}=x_{n 1}+x_{n 2}, x_{n 1} \in \operatorname{ker} A, x_{n 2} \in \operatorname{im} A$, and $Q_{A}: H \rightarrow \operatorname{im} A$, $P_{A}: H \rightarrow \operatorname{ker} A$ are the orthogonal projections such that $P_{A}+Q_{A}=\mathbb{I}$.

Since $\left(\varepsilon_{n} \mathbb{I}+A\right) / \operatorname{im} A \rightarrow \operatorname{im} A$ is invertible, we obtain

$$
\begin{gathered}
\left|x_{n 2}\right| \leq c\left|x_{n 1}+x_{n 2}\right|^{2}=c\left|x_{n 1}\right|^{2}+c\left|x_{n 2}\right|^{2}, \\
\left|x_{n 2}\right|=O\left(\left|x_{n 1}\right|^{2}\right) \quad \text { as } x_{n 1} \rightarrow 0 .
\end{gathered}
$$

We put $x_{n 1}=r_{n} \varphi_{n}, r_{n}>0, \varphi_{n} \in \partial B_{1}$. So $\left|x_{n 2}\right|=O\left(r_{n}^{2}\right)$ as $r_{n} \rightarrow 0_{+}$and

$$
\varepsilon_{n} r_{n} \varphi_{n}+P_{A} S\left(r_{n} \varphi_{n}+x_{n 2}\right)=0 \text {. }
$$

This implies

$$
\varepsilon_{n} r_{n}\left|\varphi_{n}\right|^{2}+\left\langle S\left(r_{n} \varphi_{n}+x_{n 2}\right), \varphi_{n}\right\rangle=0
$$

and so

$$
\varepsilon_{n} r_{n}+r_{n}^{\alpha}\left\langle S\left(r_{n} \varphi_{n}+x_{n 2}\right), \varphi_{n}\right\rangle / r_{n}^{\alpha}=0 .
$$

But $\left(C_{+}\right)$implies, for $n$ sufficiently large,

$$
0=\varepsilon_{n} r_{n}+r_{n}^{\alpha}\left\langle S\left(r_{n} \varphi_{n}+x_{n 2}\right), \varphi_{n}\right\rangle / r_{n}^{\alpha}>\varepsilon_{n} r_{n} .
$$

This contradiction finishes the proof.

Remark 2.3. If $S=S_{1}+S_{2}$, where $S_{1}(t x)=t^{\alpha} S_{1}(x)$ for all $t>0$ and $x \in H, S_{1}$ is continuous, $\alpha>0$ is a constant and $\left|S_{2}(x)\right|=o\left(|x|^{\alpha}\right)$ for small $x$, then the condition $\left(C_{+}\right)$, respectively $\left(C_{-}\right)$, is satisfied provided $\left\langle S_{1}(\varphi), \varphi\right\rangle>0$, respectively $\left\langle S_{1}(\varphi), \varphi\right\rangle<0$, for all $\varphi \in \partial B_{1}$.

Definition 2.4. $f \in C^{1}(H, \mathbb{R})$ is said to satisfy the condition $\left(H_{+}\right)$, respectively $\left(H_{-}\right)$, if: 
(i) $f(u)=\frac{1}{2}\langle L u, u\rangle+g(u)$, where $L: H \rightarrow H$ is a Fredholm, self-adjoint bounded linear operator for which the Morse index exists;

(ii) There is a constant $M>0$ such that $|\operatorname{grad} g(x)| \leq M$ for all $x \in H$;

(iii) $\operatorname{grad} g$ is a compact operator; that

(iv) There is a continuous map $\omega: S_{1}=\{v \in \operatorname{ker} L|| v \mid=1\} \rightarrow H$ such

$$
\lim _{r \rightarrow \infty} \operatorname{grad} g(u+r v)=\omega(v)
$$

uniformly in both $v \in S_{1}$ and $u \in \mathcal{A}$ for any fixed bounded subset $\mathcal{A}$ of $H_{1}$;

(v) There is no solution of the equation $\lambda v+P \omega(v)=0$ with $v \in S_{1}$ and $\lambda \geq 0$, respectively $\lambda \leq 0$.

THEOREM 2.5. If $f$ satisfies the condition $\left(H_{+}\right)$, respectively $\left(H_{-}\right)$, then there is a bounded neighbourhood $V$ of 0 and a constant $\varepsilon_{1}>0$ such that there are no critical points of $f(x)+\varepsilon|x|^{2} / 2$ in $H \backslash V$ for $\varepsilon_{1}>\varepsilon>0$, respectively $-\varepsilon_{1}<\varepsilon<0$.

Proof. Let $f$ satisfy $\left(H_{+}\right)$; the other case is similar. Set $f_{\varepsilon}(u)=f(u)+$ $\varepsilon|u|^{2} / 2$. Assume that there are $H \ni u_{\varepsilon_{i}} \rightarrow \infty$ such that $\varepsilon_{i} \rightarrow 0$ as $i \rightarrow \infty$ and $\operatorname{grad} f_{\varepsilon_{i}}\left(u_{\varepsilon_{i}}\right)=0$.

We take the orthogonal projections $P: H \rightarrow \operatorname{ker} L$ and $Q=\mathbb{I}-P$. Then

$$
\begin{aligned}
& \left(L+\varepsilon_{i} \mathbb{I}\right) u_{\varepsilon_{i} 2}+Q \operatorname{grad} g\left(u_{\varepsilon_{i}}\right)=0, \\
& \varepsilon_{i} u_{\varepsilon_{i} 1}+P \operatorname{grad} g\left(u_{\varepsilon_{i} 1}+u_{\varepsilon_{i} 2}\right)=0, \\
& u_{\varepsilon_{i}}=u_{\varepsilon_{i} 1}+u_{\varepsilon_{i} 2}, \quad u_{\varepsilon_{i} 1} \in \operatorname{ker} L, u_{\varepsilon_{i} 2} \in H_{1} .
\end{aligned}
$$

The assumption (ii) implies the boundedness of $\left\{u_{\varepsilon_{i} 2}\right\}$ and $\left\{\varepsilon_{i} u_{\varepsilon_{i} 1}\right\}$. So $u_{\varepsilon_{i} 1} \rightarrow \infty$. By putting $w_{\varepsilon_{i}}=u_{\varepsilon_{i} 1} /\left|u_{\varepsilon_{i} 1}\right|$ we have

$$
\varepsilon_{i}\left|u_{\varepsilon_{i} 1}\right| w_{\varepsilon_{i}}+P \operatorname{grad} g\left(u_{\varepsilon_{i} 2}+\left|u_{\varepsilon_{i} 1}\right| w_{\varepsilon_{i}}\right)=0 .
$$

We can assume $w_{\varepsilon_{i}} \rightarrow w_{0}$ and $\varepsilon_{i}\left|u_{\varepsilon_{i} 1}\right| \rightarrow \lambda_{0}$. Finally, we arrive at the equation

$$
\lambda_{0} w_{0}+P \omega\left(w_{0}\right)=0
$$

for some $w_{0} \in S_{1}$ and $\lambda_{0} \geq 0$. The contradiction with the assumption (v) proves the result.

THEOREM 2.6. If one of the following assumptions holds:

(i) $f$ satisfies $\left(C_{+}\right),\left(H_{+}\right)$and $\operatorname{index} A \neq \operatorname{index} L$;

(ii) $f$ satisfies $\left(C_{+}\right),\left(H_{-}\right)$and index $A \neq \operatorname{index} L+\operatorname{dim} \operatorname{ker} L$;

(iii) $f$ satisfies $\left(C_{-}\right),\left(H_{+}\right)$and index $A+\operatorname{dim} \operatorname{ker} A \neq \operatorname{index} L$;

(iv) $f$ satisfies $\left(C_{-}\right),\left(H_{-}\right)$and $\operatorname{index} A+\operatorname{dim} \operatorname{ker} A \neq \operatorname{index} L+\operatorname{dim} \operatorname{ker} L$, then $f$ has a critical point different from 0 .

Proof. Take $f_{\varepsilon}(x)=f(x)+\varepsilon \psi\left(|x|^{2} / 2\right)$, where

(a) $\psi(x)=x$ and $\varepsilon>0$ for the case (i); 
(b) $\psi(x)=x$ and $\varepsilon<0$ for the case (iv);

(c) For the case (iii), $\varepsilon>0$ and $\psi \in C^{\infty}(\mathbb{R}, \mathbb{R})$ is such that

$$
\psi(x)= \begin{cases}-x & \text { for }|x| \leq 1 \\ x & \text { for }|x| \geq 2\end{cases}
$$

(d) $\varepsilon<0$ and $\psi$ is as in (c) for the case (ii).

Then we take $U, V$ from Theorems 2.2 and 2.5 with $U$ shrunk and $V$ enlarged if necessary. On the other hand, we have $\operatorname{grad} f_{\varepsilon}(0)=0$ and index Hess $f_{\varepsilon}(0) \neq$ index $L_{\varepsilon}$, where $L_{\varepsilon}$ is the asymptote of grad $f_{\varepsilon}$ at infinity. Moreover, Hess $f_{\varepsilon}(0)$ and $L_{\varepsilon}$ are nondegenerate. So by the well-known result [1], there is a critical point $u_{\varepsilon}$ of $f_{\varepsilon}$ for any $\varepsilon$ small such that $u_{\varepsilon} \in V \backslash U$. Since $u_{\varepsilon} \in V$ and $V$ is bounded, we can assume that $u_{\varepsilon_{i}}$ weakly tends to some $u_{0} \in H$ as $\varepsilon_{i} \rightarrow 0$ and $i \rightarrow \infty$. By (i) and (iii) of Definition 2.4 it follows immediately that we can assume $u_{\varepsilon_{i_{j}}} \rightarrow u_{0}$ in $H$ as $j \rightarrow \infty$. Hence $u_{0} \in \bar{V} \backslash U$ is the desired critical point of $f$.

Remark 2.7. The assumption (v) of Definition 2.4 is satisfied if we suppose

(vi) For any $v \in \operatorname{ker} L$ with $|v|=1$ there is a symmetric positive semidefinite matrix $M_{v} \in \mathcal{L}(\operatorname{ker} L)$, with respect to the inner product on $H$, such that $\left\langle\omega(v), M_{v} v\right\rangle>0$, respectively $\left\langle\omega(v), M_{v} v\right\rangle<0$.

If there is an orthogonal basis $\left\{e_{i}\right\}$ of ker $L$ such that for each $v \in \operatorname{ker} L$ with $|v|=1$ there is $i$ such that $\left\langle\omega(v), v_{i}\right\rangle>0$, respectively $\left\langle\omega(v), v_{i}\right\rangle<0$, where $v=\left(v_{j}\right)$ is the orthogonal decomposition (the coordinates) of $v$ corresponding to $\left\{e_{i}\right\}$, then, by taking $M_{v} w=w_{i}$ for $w \in \operatorname{ker} L$, the assumption (vi) holds.

Of course, (vi) holds provided that we assume

$$
\langle\omega(v), v\rangle>0, \quad \text { respectively }\langle\omega(v), v\rangle<0, \quad \forall v \in \operatorname{ker} L,|v|=1 .
$$

Moreover, according to the proof of Theorem 2.5, the assumptions (iv) and (v) of Definition 2.4 can be replaced by

(vii) There is a continuous function $\widetilde{\omega}: S_{1}=\{v \in \operatorname{ker} L|| v \mid=1\} \rightarrow \mathbb{R}$ such that $\widetilde{\omega}>0$, respectively $\widetilde{\omega}<0$, on $S_{1}$ as well as

$$
\liminf _{r \rightarrow \infty}\langle\operatorname{grad} g(u+r \cdot v), v\rangle \geq \widetilde{\omega}(v),
$$

respectively

$$
\limsup _{r \rightarrow \infty}\langle\operatorname{grad} g(u+r v), v\rangle \leq \widetilde{\omega}(v),
$$

uniformly in both $v \in S_{1}$ and $u \in \mathcal{A}$ for any fixed bounded subset $\mathcal{A}$ of $H_{1}$.

The following result is a generalization of [5, Theorem 1.3]. 
TheOREm 2.8. Let $f \in C^{1}(H, \mathbb{R})$ satisfy the condition $\left(H_{+}\right)$, respectively $\left(H_{-}\right)$. Furthermore, assume that $f$ has critical points $x_{1}, \ldots, x_{k}, f$ is $C^{2}$-smooth near these points and Hess $f\left(x_{i}\right), i=1, \ldots, k$, are all Fredholm operators possessing Morse indices. If

index $L \notin\left[\operatorname{index~Hess~} f\left(x_{i}\right)\right.$, index Hess $\left.f\left(x_{i}\right)+\operatorname{dim} \operatorname{ker} H \operatorname{Hess} f\left(x_{i}\right)\right]$,

respectively

index $L+\operatorname{dim} \operatorname{ker} L$

$$
\notin\left[\operatorname{index~Hess~} f\left(x_{i}\right) \text {, index Hess } f\left(x_{i}\right)+\operatorname{dim} \operatorname{ker} \operatorname{Hess} f\left(x_{i}\right)\right] \text {, }
$$

for all $i=1, \ldots, k$, then $f$ has another critical point.

Pr o of. We consider the case $\left(H_{+}\right)$; the other one is similar. We take the perturbation

$$
g(x)=f(x)+\langle a, x\rangle+\varepsilon \widetilde{\psi}(x),
$$

where $a \in H$ and $\varepsilon>0$ are small and $\widetilde{\psi} \in C^{1}(H, \mathbb{R})$ is a convex function equal to 0 in an open neighbourhood $\mathcal{U} \subset H$ of $\left\{x_{1}, \ldots, x_{k}\right\}$ and $\widetilde{\psi}(z)=$ $|z|^{2} / 2$ for any $z$ sufficiently large. Moreover, we can assume that $\operatorname{grad} \widetilde{\psi}(B)$ is bounded for any bounded subset $B \subset H$. Such a function $\widetilde{\psi}$ can be defined by the formula $\widetilde{\psi}(z)=\tau\left(|z|^{2} / 2\right)$ for a function $\tau \in C^{\infty}(\mathbb{R}, \mathbb{R})$ such that $\tau^{\prime} \geq 0, \tau^{\prime \prime} \geq 0$ and

$$
\tau(x)= \begin{cases}0 & \text { for }|x| \leq \max _{1 \leq i \leq k}\left|x_{i}\right|^{2} / 2+1 \\ x & \text { for }|x| \geq \max _{1 \leq i \leq k}\left|x_{i}\right|^{2} / 2+3\end{cases}
$$

Of course, such a $\tau$ exists.

Let $U_{i}, i=1, \ldots, k$, be small, open bounded neighbourhoods of the points $x_{1}, \ldots, x_{k}$, respectively, such that $f$ is $C^{2}$-smooth on $\bigcup_{i=1}^{k} U_{i}$ and $\bigcup_{i=1}^{k} U_{i} \subset \mathcal{U}$. We show the existence of a critical point of $g$ in $\mathcal{B} \backslash \bigcup_{i=1}^{k} U_{i}$, where $\mathcal{B}$ is a sufficiently large, fixed ball. Indeed, if $g$ has critical points only in $\bigcup_{i=1}^{k} U_{i}$, then by applying the Sard theorem, all the critical points can be assumed to be nondegenerate. Moreover, since $a$ is small and so are the $U_{i}$, it follows that Hess $g(u)$, for any critical point $u \in U_{i}$, is Fredholm with Morse index in the interval

$$
\text { [index Hess } f\left(x_{i}\right) \text {, index Hess } f\left(x_{i}\right)+\operatorname{dim} \operatorname{ker} \operatorname{Hess} f\left(x_{i}\right) \text { ]. }
$$

This holds for all $i=1, \ldots, k$. By assumption, index $L$ is not in this interval. This contradicts [5, Theorem 1.3], since the asymptote of $\operatorname{grad} g$ is $L+\varepsilon \mathbb{I}$ and index $(L+\varepsilon \mathbb{I})=\operatorname{index} L$ for $\varepsilon>0$ small. So $g$ has a critical point in $\mathcal{B} \backslash \bigcup_{i=1}^{k} U_{i}$. By passing to the limit $a \rightarrow 0, \varepsilon \rightarrow 0_{+}$, the proof is finished as for Theorem 2.6. 
The following theorem extends the above results when there are no relations between the Morse indices of given nondegenerate critical points and the Morse indices of linear asymptotes.

TheOREM 2.9. Let $f \in C^{1}(H, \mathbb{R})$ satisfy the assumptions (i) and (iii) of Definition 2.4 with $L$ invertible and $\operatorname{grad} g(x)=o(|x|)$ as $|x| \rightarrow \infty$. Furthermore, assume that $f$ has nondegenerate critical points $x_{1}, \ldots, x_{k}$, $k \geq 2$, for which the Morse indices exist and $f\left(x_{1}\right)=\ldots=f\left(x_{k}\right)$. Then $f$ has another critical point.

Proof. If $f$ only has the critical points $x_{1}, \ldots, x_{k}$ then we take

$$
B_{K}=\{x \in H|| x \mid \leq K\},
$$

for a fixed large number $K>0$ such that

$$
\left\{x_{1}, \ldots, x_{k}\right\} \subset B_{K}, \quad h\left(B_{K}\right)=t^{\text {index } L},
$$

where $h$ denotes the generalized Morse-Conley index (see [1, p. 6 and Theorem 2.8]). Note that this index $h$ exists, since $f$ satisfies the P.S. condition. Because $f\left(x_{1}\right)=\ldots=f\left(x_{k}\right)$, the maximal invariant set in $B_{K}$ is $\left\{x_{1}, \ldots, x_{k}\right\}$. Hence (see [1, Theorem 1.1])

$$
h\left(B_{K}\right)=h\left(\left\{x_{1}, \ldots, x_{k}\right\}\right)=\sum_{i=1}^{k} h\left(\left\{x_{i}\right\}\right) .
$$

Thus

$$
t^{\operatorname{index} L}=\sum_{i=1}^{k} t^{\text {index } \operatorname{Hess} f\left(x_{i}\right)} .
$$

We obtain a contradiction. The proof is finished.

Now we give a resonant case of Theorem 2.9.

TheOREM 2.10. Let $f$ satisfy either the condition $\left(H_{+}\right)$or $\left(H_{-}\right)$. Furthermore, assume that $f$ has nondegenerate critical points $x_{1}, \ldots, x_{k}, k \geq 2$, for which the Morse indices exist and $f\left(x_{1}\right)=\ldots=f\left(x_{k}\right)$. Then $f$ has another critical point.

Proof. We consider the case $\left(H_{+}\right)$; the other one is similar. We take $f_{\varepsilon}=f+\varepsilon \widetilde{\psi}$ with $\varepsilon>0$ sufficiently small, where $\widetilde{\psi}$ is from the proof of Theorem 2.8. It is clear that $x_{1}, \ldots, x_{k}$ are the only critical points of $f_{\varepsilon}$ in some open set $\mathcal{U}_{1} \subset \mathcal{U}$ which are nondegenerate. Moreover, grad $f_{\varepsilon}$ has the linear asymptote $L+\varepsilon \mathbb{I}$ at infinity. Now we apply Theorem 2.9 to obtain a critical point of $f_{\varepsilon}$ in the set $\mathcal{B} \backslash \mathcal{U}_{1}$, where $\mathcal{B}$ is a sufficiently large, fixed ball. By passing to the limit $\varepsilon \rightarrow 0_{+}$as in the proof of Theorem 2.8, we find a nontrivial critical point of $f$. The proof is finished. 
The assumption of Theorems 2.9 and 2.10 , namely that $f$ has nondegenerate critical points $x_{1}, \ldots, x_{k}, k \geq 2$, for which the Morse indices exist and $f\left(x_{1}\right)=\ldots=f\left(x_{k}\right)$, may be naturally satisfied when $f$ is symmetric. More precisely, let $\mathcal{T}=\left\{T_{g}\right\}_{g \in \mathcal{G}}$ be a linear representation of a finite group $\mathcal{G}$ in $H$. Then $f$ is said to be invariant under $\mathcal{T}$ if

$$
f\left(T_{g} x\right)=f(x) \quad \forall(x, g) \in H \times \mathcal{G} .
$$

It is clear that if $x$ is a critical point of $f$ then $T_{g} x, g \in \mathcal{G}$, are all critical points as well. Now, $\mathcal{T}$ consists of invertible linear transformations and if $x$ is a critical point of $f$ possessing $\operatorname{Hess} f(x)$ then

$$
\text { Hess } f\left(T_{g} x\right)=\left(T_{g}^{-1}\right)^{*} \circ \operatorname{Hess} f(x) \circ T_{g}^{-1} \quad \forall g \in \mathcal{G} .
$$

Hence we see that if $x$ is nondegenerate then so is $T_{g} x$ for any $g \in \mathcal{G}$. Here * is the transpose. So by Theorems $2.9-10$ we obtain the following result.

THEOREM 2.11. Let $f \in C^{1}(H, \mathbb{R})$ satisfy either the assumptions (i) and (iii) of Definition 2.4 with $L$ invertible and $\operatorname{grad} g(x)=o(|x|)$ as $|x| \rightarrow \infty$, or $\left(H_{+}\right)$, or $\left(H_{-}\right)$. Furthermore, assume that $f$ is invariant under $\mathcal{T}$. If $f$ has a nondegenerate critical point $x$ such that $T_{g_{0}} x \neq x$ for some $g_{0} \in \mathcal{G}$, then $f$ has another critical point different from $T_{g} x, g \in \mathcal{G}$.

Remark 2.12. If there is a critical point $x$ of $f \in C^{1}(H, \mathbb{R})$, which is asymptotically quadratic and invariant under $\mathcal{T}$, such that $T_{g} x=x$ for all $g \in \mathcal{G}$ then we can restrict the study of the existence of another critical point to the space

$$
H_{s} \equiv\left\{u \in H \mid T_{g} u=u \forall g \in \mathcal{G}\right\} .
$$

The Hilbert space $H_{s}$ is invariant for $\operatorname{grad} f$, and $x \in H_{s}$ is a critical point of $f \mid H_{s}$.

We end this section with the following result.

Theorem 2.13. Let $f$ satisfy the condition $\left(H_{-}\right)$. If $f$ has a local minimum and index $L=0, \operatorname{dim} \operatorname{ker} L>0$ then $f$ has another critical point.

Proof. We assume that 0 is the given critical point and that it is a unique critical point of $f$. Take $f_{\varepsilon}=f+\varepsilon \widetilde{\psi}, \varepsilon<0$, from the proof of Theorem 2.8. So there is an open bounded neighbourhood $U$ of 0 such that $\widetilde{\psi} \mid U=0$ and the only critical point of $f$ in $U$ is 0 . Hence 0 is also the only critical point of $f_{\varepsilon}$ in $U$. Since 0 is a local minimum of $f_{\varepsilon}$, by [1, Theorem $1.1(\mathrm{v})]$, the generalized Morse-Conley index of 0 is 1 . On the other hand, this index is $t^{\text {dimker } L}$ for a large ball. This contradiction implies the existence of a critical point of $f_{\varepsilon}$ outside $U$. By passing to the limit $\varepsilon \rightarrow 0_{+}$as for Theorem 2.8, the proof is finished. 
Re mark 2.14. It follows from the proof of Theorem 2.13 that if $f$ satisfies either $\left(H_{+}\right)$or $\left(H_{-}\right)$with index $L>0$, $\operatorname{dim} \operatorname{ker} L \geq 0$ and $f$ has a local minimum, then $f$ has another critical point.

3. Weak solutions of elliptic equations. By putting

$$
H=W^{1,2}(\Omega), \quad \widetilde{H}(u, x)=\int_{0}^{u} h(v, x) d v
$$

and

$$
f(u)=\int_{\Omega}\left(\frac{1}{2}|\nabla u|^{2}+\widetilde{H}(u, x)\right) d x,
$$

we see that critical points of (3.1) are weak solutions of (1.1). The norm and inner product on $H$ are denoted by $|\cdot|_{W^{1,2}}$ and $(\cdot, \cdot)_{W^{1,2}}$, respectively. Note that $|u|_{W^{1,2}}=\sqrt{\int_{\Omega}|\nabla u|^{2} d x}$. Let $\lambda_{i}$, with $\lambda_{i+1}>\lambda_{i}>0, i=1,2, \ldots$, be the eigenvalues of $-\Delta u, u \mid \partial \Omega=0$. We put $\Sigma=\left\{-\lambda_{i}\right\}_{i=1}^{\infty}$.

Definition 3.1. $h$ is said to satisfy the condition $\left(A 1_{+}\right)$, respectively $\left(A 1_{-}\right)$, if there is a constant $q \in \Sigma$ and a function $\phi \in C(\mathbb{R} \times \bar{\Omega}, \mathbb{R})$ such that

$$
h(u, x)=q u+\phi(u, x), \quad \limsup _{|u| \rightarrow \infty}\left|\phi(u, x) / u^{4}\right|<\infty,
$$

$\lim _{u \rightarrow 0} \phi(u, x) / u^{3}=d>0, \quad$ respectively $\quad<0, \quad$ uniformly in $x \in \Omega$.

DEFINITION 3.2. $h$ is said to satisfy the condition $\left(A 2_{+}\right)$, respectively $\left(A 2_{-}\right)$, if there is a constant $p \in \Sigma$ and functions $\gamma_{ \pm} \in C(\bar{\Omega}, \mathbb{R})$ such that

$$
\lim _{u \rightarrow \pm \infty}(h(u, x)-p u)=\gamma_{ \pm}(x) \quad \text { uniformly in } x \in \Omega
$$

and

$$
\begin{aligned}
\int_{\Omega \cap\{\eta \geq 0\}} \gamma_{+} \eta d x+\int_{\Omega \cap\{\eta \leq 0\}} \gamma_{-} \eta d x & >0, \quad \text { respectively }<0, \\
\forall \eta & \in\left\{\varphi \in W^{1,2}(\Omega) \mid \Delta \varphi=p \varphi\right\} \backslash\{0\} .
\end{aligned}
$$

Theorem 3.3. If $f$ satisfies $\left(A 1_{ \pm}\right)$, respectively $\left(A 2_{ \pm}\right)$, then $f$ given by (3.1) satisfies $\left(C_{ \pm}\right)$, respectively $\left(H_{ \pm}\right)$when (iv) and $(\mathrm{v})$ of Definition 2.4 are replaced by (vii) of Remark 2.7 .

P r o of. To prove the first part of this theorem, we apply Remark 2.3 by putting

$$
\begin{aligned}
& \left(S_{1}(u), v\right)_{W^{1,2}}=d \int_{\Omega} u^{3} v d x \\
& \left(S_{2}(u), v\right)_{W^{1,2}}=\int_{\Omega}\left(\phi(u, x)-d u^{3}\right) v d x .
\end{aligned}
$$


We show that in this case $\alpha=3$. The homogeneity of $S_{1}$ in Remark 2.3 is clear for this case. We note that $W^{1,2}(\Omega) \subset L^{6}(\Omega)$ by the Sobolev imbedding theorem. Our assumptions imply that for any $r>0$ there is a constant $a=a(r)>0$ such that

$$
\left|\phi(u, x)-d u^{3}\right| \leq r|u|^{3}+a|u|^{4} \quad \forall(u, x) \in \mathbb{R} \times \Omega .
$$

So we obtain

$$
\begin{aligned}
\left|\int_{\Omega}\left(\phi(u, x)-d u^{3}\right) v d x\right| \leq & \int_{\Omega}\left(r|u|^{3}|v|+a u^{4}|v|\right) d x \\
\leq & r \sqrt{\int_{\Omega} u^{6} d x} \sqrt{\int_{\Omega} v^{2} d x} \\
& +a\left(\int_{\Omega} u^{6} d x\right)^{2 / 3}\left(\int_{\Omega}|v|^{3} d x\right)^{1 / 3} \\
\leq & c r|\nabla u|_{L^{2}}^{3}|v|_{L^{2}}+a c|\nabla u|_{L^{2}}^{4}|\nabla v|_{L^{2}} \\
\leq & \left(c r \lambda_{1}|\nabla u|_{L^{2}}^{3}+a c|\nabla u|_{L^{2}}^{4}\right)|\nabla v|_{L^{2}},
\end{aligned}
$$

for a constant $c>0$. These estimates imply that for any $r>0$ there is a constant $a_{1}=a_{1}(r)>0$ such that

$$
\left|\left(S_{2}(u), v\right)_{W^{1,2}}\right| \leq\left(r|u|_{W^{1,2}}^{3}+a_{1}|u|_{W^{1,2}}^{4}\right)|v|_{W^{1,2}} .
$$

So

$$
\left|S_{2}(u)\right|_{W^{1,2}} \leq r|u|_{W^{1,2}}^{3}+a_{1}|u|_{W^{1,2}}^{4} .
$$

Hence $\left|S_{2}(u)\right|_{W^{1,2}}=o\left(|u|_{W^{1,2}}^{3}\right)$ as $u \rightarrow 0$.

Finally, we have $\left(S_{1}(\varphi), \varphi\right)_{W^{1,2}}=d \int_{\Omega} \varphi^{4} d x$, so the assumption of Remark 2.3, $\left\langle S_{1}(\varphi), \varphi\right\rangle>0$, respectively $\left\langle S_{1}(\varphi), \varphi\right\rangle<0$, for $\varphi \in \partial B_{1}$ is satisfied as well.

To prove the second part, we put

$$
\begin{gathered}
(L v, w)_{W^{1,2}}=\int_{\Omega}(\nabla v \nabla w+p v w) d x, \\
\operatorname{ker} L=\operatorname{span}\left\{\varphi \in W^{1,2}(\Omega) \mid \Delta \varphi=p \varphi\right\} .
\end{gathered}
$$

So we get (see [7, Theorem 3.5.94])

$$
\widetilde{\omega}(\eta)=\int_{\Omega \cap\{\eta \geq 0\}} \gamma_{+} \eta d x+\int_{\Omega \cap\{\eta \leq 0\}} \gamma_{-} \eta d x
$$

for any $\eta \in \operatorname{ker} L$ such that $|u|_{W^{1,2}}=1$. Hence the condition (vii) of Remark 2.7 holds.

THEOREM 3.4. If one of the following conditions is satisfied:

(a) $h$ satisfies $\left(A 1_{+}\right),\left(A 2_{+}\right)$and $p \neq q$; 

$\neq \emptyset$

(b) $h$ satisfies $\left(A 1_{+}\right),\left(A 2_{-}\right)$and either $p \leq q$, or $p>q$ with $(q, p) \cap \Sigma$

(c) $h$ satisfies $\left(A 1_{-}\right),\left(A 2_{+}\right)$and either $p \geq q$, or $p<q$ with $(p, q) \cap \Sigma \neq \emptyset$;

(d) $h$ satisfies $\left(A 1_{-}\right),\left(A 2_{-}\right)$and $p \neq q$,

then (1.1) has a nonzero weak solution.

Proof. By using Theorem 3.3 and

$$
\begin{aligned}
\operatorname{index} A & =\operatorname{dim} \operatorname{span}\left\{\varphi \in W^{1,2}(\Omega) \mid-\Delta \varphi=\lambda \varphi, q<-\lambda\right\}, \\
\operatorname{dim} \operatorname{ker} A & =\operatorname{dim} \operatorname{span}\left\{\varphi \in W^{1,2}(\Omega) \mid-\Delta \varphi=\lambda \varphi, q=-\lambda\right\}, \\
\operatorname{index} L & =\operatorname{dim} \operatorname{span}\left\{\varphi \in W^{1,2}(\Omega) \mid-\Delta \varphi=\lambda \varphi, p<-\lambda\right\}, \\
\operatorname{dim} \operatorname{ker} L & =\operatorname{dim} \operatorname{span}\left\{\varphi \in W^{1,2}(\Omega) \mid-\Delta \varphi=\lambda \varphi, p=-\lambda\right\},
\end{aligned}
$$

the result follows by Theorem 2.6.

Now we deal with cases when $h$ has a symmetry.

Theorem 3.5. Consider (1.1) with $-\Omega=\Omega$ and suppose $h$ is $C^{1}$-smooth in $u$ such that $\frac{\partial}{\partial u} h(\cdot, \cdot)$ is uniformly bounded on $\mathbb{R} \times \Omega$ and

$$
h(u, x)=h(u,-x) \quad \forall(u, x) \in \mathbb{R} \times \Omega .
$$

Assume that $f$ satisfies either the condition $\left(A 2_{+}\right)$or $\left(A 2_{-}\right)$. If there is a weak solution $v$ of (1.1) such that $v(x) \neq v(-x)$ on a subset of $\Omega$ with a nonzero Lebesgue measure and

$$
\left[\inf _{\Omega} \frac{\partial}{\partial u} h(v(x), x), \sup _{\Omega} \frac{\partial}{\partial u} h(v(x), x)\right] \cap \Sigma=\emptyset,
$$

then (1.1) has at least 3 weak solutions.

Proof. We apply Theorem 2.11 in the proof of Theorem 3.4. Now $\mathcal{G}=$ $\mathbb{Z}_{2} \equiv\{0,1\}$ and

$$
\left(T_{1} u\right)(x) \equiv u(-x) \quad \forall x \in \Omega .
$$

The assumptions imply that $v$ is a nondegenerate critical point of $f$, where $f$ is given by (3.1), and has a Morse index. Hence (1.1) has at least 3 weak solutions: $v(x), v(-x)$ and the one predicted by Theorem 2.11 .

R e m a r k 3.6. Consider (1.1) with $h$ satisfying the conditions of Theorem 3.5 and having a weak and symmetric solution $v$, i.e. $v(x)=v(-x)$ for almost all $x \in \Omega$. By applying Remark 2.12 in the proof of Theorem 3.5, we can find another weak and symmetric solution of (1.1). We note that now the set $\Sigma$ is replaced by

$$
\Sigma_{e}=\left\{\lambda \in \mathbb{R} \mid \Delta \varphi=\lambda \varphi, 0 \neq \varphi \in W^{1,2}(\Omega), \varphi(x)=\varphi(-x) \forall x \in \Omega\right\},
$$

since the space $H_{s}$ of Remark 2.12 has the form

$$
H_{e}=\left\{\varphi \in W^{1,2}(\Omega) \mid \varphi(x)=\varphi(-x) \forall x \in \Omega\right\} .
$$


Re mark 3.7. The symmetry of $h$ in Theorem 3.5 could be replaced by

$$
h(u, x)=-h(-u,-x) \quad \forall(u, x) \in \mathbb{R} \times \Omega .
$$

Then we can modify Theorem 3.5 and Remark 3.6 for this case by taking $\mathcal{G}=\mathbb{Z}_{2}$ and

$$
\left(T_{1} u\right)(x) \equiv-u(-x) \quad \forall x \in \Omega .
$$

We note that now the set $\Sigma$ is replaced by

$$
\Sigma_{0}=\left\{\lambda \in \mathbb{R} \mid \Delta \varphi=\lambda \varphi, 0 \neq \varphi \in W^{1,2}(\Omega), \varphi(x)=-\varphi(-x) \forall x \in \Omega\right\},
$$

since the space $H_{s}$ of Remark 2.12 has the form

$$
H_{0}=\left\{\varphi \in W^{1,2}(\Omega) \mid \varphi(x)=-\varphi(-x) \forall x \in \Omega\right\} .
$$

Now we apply Theorem 2.8.

TheOrem 3.8. Assume $h$ is $C^{1}$-smooth in $u, \frac{\partial}{\partial u} h(\cdot, \cdot)$ is uniformly bounded on $\mathbb{R} \times \Omega$ and $\lim _{u \rightarrow 0} h(u, x) / u=q \in \Sigma$ uniformly in $x \in \Omega$. If one of the following conditions is satisfied:

(i) $h$ satisfies $\left(A 2_{+}\right)$and either $q<p$, or $q>p$ with $(p, q) \cap \Sigma \neq \emptyset$;

(ii) $h$ satisfies $\left(A 2_{-}\right)$and either $q>p$, or $q<p$ with $(q, p) \cap \Sigma \neq \emptyset$,

then $f$ has a nonzero weak solution.

Proof. We have

$$
\begin{aligned}
\operatorname{index} A & =\operatorname{dim} \operatorname{span}\left\{\varphi \in W^{1,2}(\Omega) \mid-\Delta \varphi=\lambda \varphi, q<-\lambda\right\}, \\
\operatorname{dim} \operatorname{ker} A & =\operatorname{dim} \operatorname{span}\left\{\varphi \in W^{1,2}(\Omega) \mid-\Delta \varphi=\lambda \varphi, q=-\lambda\right\}, \\
\operatorname{index} L & =\operatorname{dim} \operatorname{span}\left\{\varphi \in W^{1,2}(\Omega) \mid-\Delta \varphi=\lambda \varphi, p<-\lambda\right\}, \\
\operatorname{dim} \operatorname{ker} L & =\operatorname{dim} \operatorname{span}\left\{\varphi \in W^{1,2}(\Omega) \mid-\Delta \varphi=\lambda \varphi, p=-\lambda\right\} .
\end{aligned}
$$

If $q>p$ and $(p, q) \cap \Sigma \neq \emptyset$ then index $L>$ index $A+\operatorname{dim} \operatorname{ker} A$. If $q<p$ then index $L<\operatorname{index} A$. If $q<p$ and $(q, p) \cap \Sigma \neq \emptyset$ then index $L+\operatorname{dim} \operatorname{ker} L<$ index $A$. If $q>p$ then index $L+\operatorname{dim} \operatorname{ker} L>\operatorname{index} A+\operatorname{dim} \operatorname{ker} A$. So the proof is finished by Theorem 2.8.

Remark 3.9. According to Theorem 3.4, if $h$ satisfies, in addition to the assumptions of Theorem 3.8, either $\left(A 1_{+}\right)$or $\left(A 1_{-}\right)$, then there are more $(p, q)$ for which (1.1) has a nonzero weak solution.

Finally, we apply Theorem 2.13 to (1.1).

Theorem 3.10. If

$$
\liminf _{u \rightarrow 0} \widetilde{H}(u, x) / u^{2}>-\lambda_{1} / 2 \quad \text { uniformly in } x \in \Omega
$$

and $h$ satisfies the condition $\left(A 2_{-}\right)$with $p=-\lambda_{1}$ then (1.1) has a nonzero weak solution. 
Proof. We apply Theorem 2.13. It is enough to show that $u=0$ is a local minimum of $f$ defined by (3.1), since now clearly index $L=0$ and $\operatorname{dim} \operatorname{ker} L>0$. By assumption, for any sufficiently small $r>0$ there is a constant $d=d(r)>0$ such that

$$
\widetilde{H}(u, x) \geq \frac{1}{2}\left(-\lambda_{1}+r\right) u^{2}-d|u|^{3} \quad \forall(u, x) \in \mathbb{R} \times \Omega .
$$

Hence by the Sobolev imbedding theorem and $\lambda_{1}>0$, we have for a small $r>0$,

$$
\begin{aligned}
f(u) & =\frac{1}{2}|\nabla u|_{L^{2}}^{2}+\int_{\Omega} \widetilde{H}(u, x) d x \geq \frac{1}{2}|\nabla u|_{L^{2}}^{2}+\frac{1}{2}\left(-\lambda_{1}+r\right)|u|_{L^{2}}^{2}-d c|\nabla u|_{L^{2}}^{3} \\
& \geq \frac{1}{2}|\nabla u|_{L^{2}}^{2}+\frac{1}{2}\left(-\lambda_{1}+r\right)|\nabla u|_{L^{2}}^{2} / \lambda_{1}-d c|\nabla u|_{L^{2}}^{3} \\
& =\frac{r}{2 \lambda_{1}}|\nabla u|_{L^{2}}^{2}-d c|\nabla u|_{L^{2}}^{3},
\end{aligned}
$$

for a constant $c>0$. So $f(u)>0$ for $u \neq 0$ sufficiently small.

4. Nonzero solutions of the problem (1.2). It is clear that in the notations of the previous section we have $h(y, x)=-n^{2} y+g(y)$ as well as $\Sigma=\left\{-i^{2} \mid i \in \mathbb{N}\right\}$ and the corresponding eigenvectors are simple.

TheOREM 4.1. $h(y, x)=-n^{2} y+g(y)$ with $\Omega=[0, \pi]$ satisfies the condition $\left(A 2_{+}\right)$, respectively $\left(A 2_{-}\right)$, if there is a $k \in \mathbb{N}$ and constants $g( \pm \infty)$ such that

$$
\lim _{y \rightarrow \pm \infty}\left(g(y)-\left(n^{2}-k^{2}\right) u\right)=g( \pm \infty)
$$

and

$$
\left\{\begin{array}{l}
g(+\infty)>0, g(-\infty)<0 \quad \text { for } k=1 \\
g(+\infty)>g(-\infty) \text { for } k=2 j, \\
g(+\infty)>\max \{j g(-\infty) /(j+1),(j+1) g(-\infty) / j\} \\
\quad \text { for } k=2 j+1, \text { where } j \in \mathbb{N}
\end{array}\right.
$$

respectively

$$
\left\{\begin{array}{l}
g(+\infty)<0, g(-\infty)>0 \quad \text { for } k=1, \\
g(+\infty)<g(-\infty) \text { for } k=2 j, \\
g(+\infty)<\min \{j g(-\infty) /(j+1),(j+1) g(-\infty) / j\} \\
\quad \text { for } k=2 j+1, \text { where } j \in \mathbb{N} .
\end{array}\right.
$$

Pro of. The conditions of Definition 3.2 now read

$$
\int_{[0, \pi] \cap\{c \sin k x \geq 0\}} g(+\infty) c \sin k x d x+\int_{[0, \pi] \cap\{c \sin k x \leq 0\}} g(-\infty) c \sin k x d x>0,
$$


respectively

$$
\int_{[0, \pi] \cap\{c \sin k x \geq 0\}} g(+\infty) c \sin k x d x+\int_{[0, \pi] \cap\{c \sin k x \leq 0\}} g(-\infty) c \sin k x d x<0
$$

for $c= \pm 1$. From the assumptions of this theorem, it is clear that they are satisfied for this case.

THEOREM 4.2. (3.1) with $h(y, x)=-n^{2} y+g(y)$ and $\Omega=[0, \pi]$ satisfies the condition $\left(C_{+}\right)$, respectively $\left(C_{-}\right)$, provided that (1.3) holds with $l_{ \pm} \geq 0$, $l_{+}^{2}+l_{-}^{2}>0$, respectively $l_{ \pm} \leq 0, l_{+}^{2}+l_{-}^{2}>0$.

Proof. We apply Remark 2.3 with

$$
\left(S_{1}(y), v\right)_{W^{1,2}}=\int_{0}^{\pi} \widetilde{g}(y) v d x, \quad\left(S_{2}(y), v\right)_{W^{1,2}}=\int_{0}^{\pi}(g(y)-\widetilde{g}(y)) v d x
$$

and

$$
\widetilde{g}(y)= \begin{cases}l_{+} y^{m} & \text { for } y \geq 0 \\ -l_{-}|y|^{m} & \text { for } y \leq 0 .\end{cases}
$$

We show that in this case $\alpha=m$. The homogeneity of $S_{1}$ in Remark 2.3 is clear for this case. Since $W^{1,2}[0, \pi] \subset C[0, \pi]$ by the Sobolev imbedding theorem and

$$
\lim _{y \rightarrow 0}(g(y)-\widetilde{g}(y)) / y^{m}=0,
$$

we see that $\left|S_{2}(y)\right|_{W^{1,2}}=o\left(|y|_{W^{1,2}}^{m}\right)$ as $y \rightarrow 0$. Finally, for $c= \pm 1$ we have $\left(S_{1}(c \sin n x), c \sin n x\right)_{W^{1,2}}$

$$
=l_{+} \int_{[0, \pi] \cap\{c \sin n x>0\}}(c \sin n x)^{m+1} d x+l_{-} \int_{[0, \pi] \cap\{c \sin n x<0\}}|c \sin n x|^{m+1} d x .
$$

So the last condition of Remark 2.3, $\left\langle S_{1}(\varphi), \varphi\right\rangle>0$, respectively $\left\langle S_{1}(\varphi), \varphi\right\rangle$ $<0$, for $\varphi \in \partial B_{1}$, is satisfied as well.

THEOREM 4.3. If one of the following assumptions holds:

(i) (4.1-2) with $n \neq k$ as well as (1.3) with $l_{ \pm} \geq 0, l_{+}^{2}+l_{-}^{2}>0$ are satisfied;

(ii) (4.1), (4.3) with $n-1 \neq k$ as well as (1.3) with $l_{ \pm} \geq 0, l_{+}^{2}+l_{-}^{2}>0$ are satisfied;

(iii) (4.1-2) with $n \neq k-1$ as well as (1.3) with $l_{ \pm} \leq 0, l_{+}^{2}+l_{-}^{2}>0$ are satisfied;

(iv) (4.1), (4.3) with $n \neq k$ as well as (1.3) with $l_{ \pm} \leq 0, l_{+}^{2}+l_{-}^{2}>0$ are satisfied,

then (1.2) has a nonzero solution.

Proof. This follows from Theorem 2.6 as in Theorem 3.4, since now index $A=n-1, \operatorname{dim} \operatorname{ker} A=1$ and index $L=k-1, \operatorname{dim} \operatorname{ker} L=1$. 
TheOREM 4.4. Consider (1.2) where instead of (1.3) we suppose that $g$ is $C^{1}$-smooth for $y$ small and $g^{\prime}(0)=0$. Assume, in addition, that either (4.1-2) hold with $k \neq n, k \neq n+1$, or (4.1), (4.3) hold with $k \neq n-1$, $k \neq n$. Then (1.2) has a nonzero solution.

Proof. As in the previous proof, this is already done in Theorem 3.8.

\section{References}

[1] V. Benci, Some applications of the generalized Morse-Conley index, Confer. Semin. Mat. Univ. Bari 218 (1987).

[2] M. Fečkan, Critical points of asymptotically quadratic functions, Ann. Polon. Math. 61 (1995), 63-76.

[3] - On a theorem of L. Lefton, Math. Slovaca 42 (1992), 195-200.

[4] L. Lefton, Existence of small solutions to a resonant boundary value problem with large nonlinearity, J. Differential Equations 85 (1990), 171-185.

[5] S. Li and J. Q. Liu, Morse theory and asymptotic linear Hamiltonian system, ibid. 78 (1989), 53-73.

[6] J. Mawhin and M. Willem, Critical Point Theory and Hamiltonian Systems, Springer, New York, 1989.

[7] J. Nečas, Introduction to the Theory of Nonlinear Elliptic Equations, Teubner, Leipzig, 1983.

[8] B. Przeradzki, An abstract version of the resonance theorem, Ann. Polon. Math. 53 (1991), 35-43.

Department of Mathematical Analysis

Faculty of Mathematics and Physics

Comenius University

Mlynská dolina

84215 Bratislava, Slovakia 